Citation: G. Bressan, A. Guaran, F. Visentin, G.P. Zaccomer (2021). Aspetti geografici del confronto fra sapere esperto e contestuale: un'analisi regionale nei paesaggi del 'degrado'. Bollettino della Società Geografica Italiana serie 14, 4(2): 3-18. doi: 10.36253/ bsgi-1350

Copyright: (c) 2021 G. Bressan, A. Guaran, F. Visentin, G.P. Zaccomer. This is an open access, peer-reviewed article published by Firenze University Press (http://www.fupress.com/bsgi) and distributed under the terms of the Creative Commons Attribution License, which permits unrestricted use, distribution, and reproduction in any medium, provided the original author and source are credited.

Data Availability Statement: All relevant data are within the paper and its Supporting Information files.

Competing Interests: The Author(s) declare(s) no conflict of interest.

The paper was conceived jointly by all the authors. For Italian evaluation purposes, Giorgia Bressan takes responsibility for sections 2.1 and 3.3, Andrea Guaran and Francesco Visentin for sections 1 and 3.2, Gian Pietro Zaccomer for sections 2.2 and 3.1; all the authors have elaborated jointly the conclusions.

\section{Aspetti geografici del confronto fra sapere esperto e contestuale: un'analisi regionale nei paesaggi del "degrado"}

\author{
Geographical aspects of the comparison between expert and \\ contextual knowledge: a regional analysis of the perceptions of \\ degraded landscapes
}

\author{
Giorgia Bressan, Andrea Guaran, Francesco Visentin, Gian Pietro \\ ZACCOMER \\ Dipartimento di Lingue e Letterature, Comunicazione, Formazione e Società, Università \\ degli Studi di Udine, Italia \\ E-mail: giorgia.bressan@uniud.it, andrea.guaran@uniud.it, francesco.visentin@uniud.it, \\ gianpietro.zaccomer@uniud.it
}

\begin{abstract}
During the last decades, the active participation of the inhabitants in the interpretation, management and planning of landscape has assumed an increasingly important role, thanks to the challenges and understandings provided by the European Landscape Convention which advocated the participation and enhanced the role of the populations as fundamental sources of information. Despite the difficulties linked to the valorization of such assets, the comparison between expert and public views can favour the production of new knowledge as long as these information and sources could be compared and connected through fruitful approaches. The present contribution finds his roots in the development of Friuli Venezia Giulia Regional Landscape Plan and, adopting a participatory approach, tried to investigate the perceived landscapes of risk and degradation. The focus is on three categories of areas and sites perceived as degraded, specifically those resulting from the massive presence of abandonment former military areas, hydrogeological problems and, finally, quarry activities. After presenting the most relevant aspects and the results of the geo-cartographic analysis, the limits and opportunities of the research are highlighted. On the one hand, similarly to other participatory projects, the analysis presents problems in terms of quality and territorial coverage, on the other hand, it offers reflections on the possible synergies between local and expert knowledge.
\end{abstract}

Keywords: degraded landscape, perception, expert and contextual knowledge, georeferencing, dataset integration.

Riassunto. Negli ultimi decenni la partecipazione attiva degli abitanti per l'interpretazione, la gestione e la pianificazione del paesaggio ha assunto un ruolo sempre più rilevante, grazie agli indirizzi proposti dalla Convenzione Europea del Paesaggio che individua l'esperienza e il sentire delle popolazioni come fondamentali fonti informative. Nonostante le difficoltà implicite nella valorizzazione di queste risorse, il confronto tra il sapere esperto e contestuale può favorire l'elaborazione di nuove conoscenze, a 
patto che si possano confrontare le informazioni e mettere in effettiva e fruttuosa comunicazione i due saperi. Il contributo si sviluppa a valle di un percorso progettuale di natura partecipativa sui paesaggi del rischio e del degrado che si inserisce nel tracciato pluriennale durante il quale è stato elaborato il Piano Paesaggistico Regionale del Friuli Venezia Giulia. Lattenzione si focalizza su tre categorie di aree e siti percepiti come degradati: quelle derivate dall'imponente ex presenza militare, dal dissesto idraulico fluviale e, infine, dalle attività di cava. Dopo aver presentato gli aspetti e risultati più salienti dell'analisi geo-cartografica, si mettono in rilievo i limiti e le opportunità della ricerca che, da un lato, analogamente ad altri progetti di geografia partecipativa, presenta problemi di qualità e di copertura territoriale, dall'altro offre riflessioni sulle possibili sinergie tra il sapere locale e quello esperto.

Parole chiave: paesaggi del degrado, percezione, sapere esperto e contestuale, georeferenziazione, integrazione dataset.

\section{Introduzione}

La conflittualità a livello territoriale in relazione alla gestione e interpretazione del paesaggio vissuto, sia a livello personale che comunitario, ha assunto sempre di più negli ultimi anni un ruolo centrale nel dibattito accademico e pubblico grazie alla diffusione e penetrazione a vari livelli e scale (geografica, sociale, amministrativa e normativa) dei dettami proposti dalla Convenzione Europea del Paesaggio (CEP). Il paesaggio può quindi essere letto ed interpretato come "l'espressione delle pratiche sociali che lo hanno costruito in passato e che continuano a produrne le attuali modificazioni" (Castiglioni et al. 2010, 94; Castiglioni, Ferrario 2020). In questo continuo processo di cambiamento, gli abitanti sono chiamati in causa non solo come fruitori ma anche in veste di attori principali tanto che nell'art. 1 della CEP si afferma che il “paesaggio' designa una determinata parte di territorio, così come è percepita dalle popolazioni, il cui carattere deriva dall'azione di fattori naturali e/o umani e dalle loro interrelazioni" (Consiglio d'Europa 2000); inoltre, all'art. 5 la CEP definisce il paesaggio come una "componente essenziale del contesto di vita delle popolazioni, espressione delle diversità" (Consiglio d'Europa 2000). Questo indirizzo e invito non ha ovviamente esaurito o ridimensionato quello che il geografo catalano Joan Nogué chiama il "conflitto di rappresentazione" $(2017,88)$, connesso al fatto che i paesaggi di "riferimento" (da intendere come le immagini più significative e tradizionalmente più rappresentative) si allontanano sempre di più da quelli del quotidiano, creando cortocircuiti percettivi e sentimentali (Vallera- ni, Varotto 2005). Questo "conflitto di rappresentazione" può anche interessare i processi di gestione, progettazione e valorizzazione del territorio, dove il confronto tra gli attori presenta ancora delle problematiche, a livello partecipativo, comunicativo e di competenze, comportando disallineamenti e incomprensioni fra i saperi e gli attori (Castiglioni, De Marchi 2009; Roe 2012; Varotto 2012; Poli 2013). Il paesaggio e la sua percezione, gestione e progettazione da parte degli abitanti, dei tecnici e dei decisori politici sono intimamente interconnessi: non è solo una questione di cornice o contesto, ma di intendere il paesaggio come una forma sensibile, interfaccia e momento di congiunzione, seppur sfumata nei suoi contorni e sempre meno nitida nei suoi limiti, tra il percepire e l'agire, tra la rappresentazione e la vivibilità, nonché una utile cartina di tornasole per cercare di comprendere i meccanismi con i quali le società si rapportano, idealmente e materialmente, ai territori (Papotti 2008; Turri 2014). Su questo punto Jean-Marc Besse parla di sfida per la “...determinazione della giusta 'misura', cioè quello dell'adeguamento tra il desiderabile e il sopportabile. Una regolazione che non può darsi al di fuori dell'esperienza sensibile e che non può essere predefinita e predeterminata da un ufficio tecnico" (2020, 31). Infatti, il patrimonio territoriale, così come esplicitato da Alberto Magnaghi (2005), necessita di una relazione sempre più stretta tra la sfera esperta, politica e tecnica, e la conoscenza locale, di cui le popolazioni sono depositarie. Queste ultime, infatti, includendo allo stesso tempo percezioni, conoscenze e progettualità individuali e la loro sintesi sul piano comunitario, sono in grado di confezionare e proporre l'insostituibile sapere contestuale (Magnaghi 2020), fondamentale per identificare i bisogni, le aspirazioni e il patrimonio di un territorio.

Proprio su questo possibile dialogo (cercato, abbozzato, mancato), e sul problematico rapporto tra la conoscenza tecnica (esperta) e quella esperienziale/spontanea (contestuale), quest'ultima definibile anche come sapere profano che caratterizza una sorta di conoscenza ordinaria (Perrone 2011), si inserisce il presente contributo, con la consapevolezza però dell'esistenza di una certa vaghezza nell'impiego di queste categorie, per certi versi dicotomiche. Al riguardo, Quaini (2007) sottolinea, relativamente al rapporto tra i due saperi, come questi termini siano usati spesso con disinvoltura in modo intercambiabile, generando una certa approssimazione, frutto dell'incapacità di attribuire significati chiari ed univoci. Forti risultano l'urgenza e la consapevolezza che la collaborazione sia sempre di più un fattore imprescindibile ai fini sia della elaborazione di linee progettuali e di pianificazione che per gli interventi gestionali, i quali incidono sui caratteri paesaggistici. Inoltre, l'azione collabora- 
tiva riafferma come la lente del paesaggio possa risultare utile alla identificazione e costruzione di un rinnovato rapporto tra i due saperi, favorendo il benessere, la soddisfazione e l'identificazione del soggetto nel paesaggio (Dematteis 2007).

Infatti, come sottolineato da Mattioli, "se dotati di informazioni adeguate, gli abitanti sono in grado di dialogare in modo costruttivo coi tecnici, dando apporti significativi al progetto ed esprimendo una ingegneria popolare" $(2012,5)$. D'altronde gli abitanti sono coloro che vivono in prima persona e direttamente i cambiamenti o le modifiche, identificando le loro figure come "testimoni di una memoria collettiva di 'lunga durata', fatta di tradizioni orali, di modalità storiche di utilizzo dello spazio, di relazioni tra le persone e tra i gruppi sociali, e come soggetti che percepiscono e rappresentano in modo diverso il proprio quadro di vita" (Balletti 2007, 14).

L'articolo si sviluppa a valle di un percorso progettuale (PaRiDe: I Paesaggi del rischio e del degrado: dalla percezione alla rappresentazione e alla territorializzazione) che si inserisce nel tracciato pluriennale durante il quale è stato elaborato il Piano Paesaggistico Regionale (Regione Autonoma FVG 2018a). Tra gli obiettivi di queste ricerche, vi era la volontà di indagare e studiare la complessità dei paesaggi del Friuli Venezia Giulia (FVG), ricorrendo a diversi strumenti e modalità per coinvolgere i cittadini e recuperare così il loro sentire più autentico, essendo consci che "il paesaggio sia da considerare come 'cartina al tornasole' di valori, del degrado, dei problemi del territorio" (Balletti 2007, 26).

I dati, ricavati mediante l'impiego di indagini puntuali tramite questionari e il ricorso ad uno specifico webGIS, hanno permesso di mettere a confronto sul piano qualitativo le segnalazioni pervenute con le coerenti banche dati disponibili, sia istituzionali sia elaborate in altre precedenti azioni di ricerca o frutto di processi di cartografia partecipata validata, come OpenStreetMap (OSM). Lo scopo dell'iniziativa, realizzata ricorrendo alla produzione di una cartografia che potesse garantire la sovrapposizione degli strati georeferenziati, è quello di verificare le possibili interazioni dei 'linguaggi' utilizzati e delle eventuali indicazioni sul terreno degli interventi concreti da mettere in atto per accrescere la conoscenza in relazione a situazioni di abbandono, trasformazioni e decadimento della qualità del paesaggio.

Quindi la scelta è ricaduta su tre categorie di alterazione e compromissione del paesaggio, in relazione ai giudizi espressi dai cittadini, che riguardano i siti e le strutture militari, i corpi idrici e le aree di cava, anche alla luce della sempre più cogente rilevanza sottolineata nel preambolo della CEP nei confronti del paesaggio quale "elemento importante della qualità della vita delle popolazioni: nelle aree urbane e nelle campagne, nei territori degradati, come in quelli di grande qualità, nelle zone considerate eccezionali, come in quelle della vita quotidiana" (Consiglio d'Europa 2000). Va precisato che l'impiego del termine "degrado" propone una indubbia difficoltà di definizione, trattandosi di un aspetto soprattutto ascrivibile ad una dimensione percettiva e come tale riconducibile alla sfera soggettiva. Tuttavia, dal momento che il Codice dei beni culturali e del paesaggio (Presidenza della Repubblica Italiana 2004) prevede specifici interventi volti alla riqualificazione delle aree compromesse o degradate (art. 143, comma 1, lettera g) e richiede che la pianificazione regionale si esprima adeguatamente in tal senso, è opportuno cercare di circoscrivere il significato dell'espressione. Così con il termine degrado è possibile fare riferimento ad un processo di alterazione dei caratteri e della qualità distintivi di un paesaggio, minandone la sua integrità sul piano percettivo e funzionale (Tondelli, Conticelli 2012).

L'operazione di affiancamento e, in un certo qual modo, di analisi comparata delle segnalazioni frutto del sapere contestuale con le corrispondenti informazioni ricavate all'interno delle fonti consultate che esprimono la conoscenza esperta e codificata, delinea il tentativo empirico di allacciare un dialogo tra i due saperi, tecnico-politico e spontaneo-locale, al fine di accrescere il patrimonio di conoscenza condivisa, da ritenersi essenziale per un migliore governo del territorio (Fischer 2020). Nel contributo, dopo aver discusso gli aspetti metodologici che sono alla base del lavoro di ricerca e presentato le fonti utilizzate, ci si sofferma sui casi studio selezionati. L'intenzione è di evidenziare i punti di contatto e le diffrazioni tra i due saperi, con l'obiettivo di sottolineare la necessità di un rinnovato e continuo dialogo, nonché le possibili aperture che una loro interazione e possibile codificazione nella raccolta dei dati (temporale, modalità di stimolo, rivisitazione della popolazione target, ecc.) potrebbero apportare nella pianificazione e gestione del paesaggio.

\section{Aspetti metodologici}

\subsection{Le fonti utilizzate}

Il sapere esperto è identificato, ai fini della ricerca, in una serie di dataset costituenti le "fonti esterne al progetto", cioè prodotte da altri soggetti per finalità conoscitive diverse da quelle di questo studio. Alcune fonti nascono come database contenenti elementi georeferenziati. Altre, invece, sono tabelle non archiviate 
in un GIS che, includendo riferimenti geografici solo in forma testuale (es: nominativo del Comune), sono state trasformate per il progetto in dati georeferenziati mediante un'operazione di geocoding. Anche fra i database prettamente spaziali bisogna distinguere varie tipologie di fonti: quelle di natura partecipativa e i dataset ufficiali di istituzioni formali locali. Non esistendo al momento un catalogo ufficiale che rappresenti il sapere esperto, tali fonti cambiano in base alla tematica e al contesto geografico in analisi. Ad ogni modo, OSM risulta il dataset geografico di natura partecipativa che, includendo allo stesso tempo elementi antropici e naturali, permette una primissima valutazione della qualità e del contenuto informativo delle segnalazioni ricevute, a prescindere dall'argomento affrontato.

Le segnalazioni dei cittadini partecipanti al progetto rappresentano in questo lavoro il sapere contestuale e, sotto un profilo operativo, costituiscono le "fonti interne al progetto". Si tratta di dataset spaziali che contengono dati originali, quindi non presenti in altri archivi, sulla percezione dei cittadini in merito all'esistenza di situazioni percepite di abbandono o degrado e di valore nel territorio regionale. Le segnalazioni risultano essere, in linea di principio, nuova conoscenza prodotta. Questi dati potrebbero sembrare simili, a causa della loro genesi partecipativa, a quelli presenti nel database del progetto collaborativo di OSM, ma ci sono diverse dissomiglianze fra i due. La principale è che mentre quest'ultimo è composto da elementi geografici oggettivamente identificabili, le segnalazioni riguardano percezioni che geograficamente possono rappresentare solo una frazione della geometria riconducibile allo stesso elemento di OSM. Così, ad esempio, solo un'area di un parco, e non l'intero parco, può apparire in stato di abbandono per un segnalatore.

Le segnalazioni sono state acquisite, in parte, tramite piattaforma webGIS, tra dicembre 2015 e maggio 2016, mediante un processo di raccolta dati che non aveva solo finalità di ricerca, ma anche concreti obiettivi di pianificazione per l'elaborazione del Piano Paesaggistico Regionale del FVG (PPR-FVG). Altri dati sono l'esito di una raccolta successiva, condotta nel contesto del già citato progetto PaRiDe, che, nel corso del 2018, ha previsto lo svolgimento di quattro indagini statistiche sulla percezione dei paesaggi regionali (Bressan, Pascolini 2019). In due indagini, basate su Google Form, il partecipante indicava in forma testuale il sito da segnalare; in una terza, lo strumento era un geo-questionario progettato nella piattaforma Enketo in cui le risposte alla domanda geografica sulle segnalazioni venivano fornite utilizzando delle mappe interattive incluse in un questionario online; infine, la quarta ha fatto uso di un modulo che permetteva il caricamento di fotografie e l'inserimento di una breve descrizione testuale del luogo fotografato. Il sapere locale geografico è stato quindi intercettato secondo diverse modalità, al fine di coinvolgere un gruppo eterogeneo di persone, con diverse conoscenze geografiche e capacità GIS. Il dato raccolto, tramite webGIS e geoquestionario, era già georeferenziato, in quanto il partecipante si trovava ad interagire con una mappa online. Negli altri casi, la georeferenziazione era un'operazione compiuta posteriormente alla fase di raccolta dati dal ricercatore, in quanto il cittadino si limitava a fornire una descrizione testuale della localizzazione del sito da segnalare. Nonostante queste differenze, le segnalazioni raccolte online sono state in tutti i cinque casi fornite da persone che volontariamente, senza supervisione e con limitate istruzioni, hanno deciso di contribuire grazie ad un invito divulgato attraverso la rete.

Dal momento che l'approccio adottato per la raccolta dati non assicura la rappresentatività, è centrale, prima di affrontare un qualsiasi confronto con fonti esterne, approfondire la distribuzione spaziale dei dati percettivi.

\subsection{Una lettura di sintesi delle segnalazioni}

Una lettura di sintesi sulla distribuzione spaziale delle 1.593 segnalazioni relative al degrado percepito, sulle 4.749 totali ricevute complessivamente nel contesto del webGIS e del progetto PaRiDe, è stata resa possibile ricorrendo a indicatori statistici riadattati per il presente studio (Guarini, Tassinari 2000). Per quanto riguarda la scala di riferimento si è deciso di adottare quella elaborata durante la redazione del PPR-FVG quando sono stati individuati 12 Ambiti di Paesaggio (AP) come da Fig. 1 (Zaccomer 2018; Guaran, Pascolini 2019). Dal punto di vita statistico, il fatto di prendere in considerazione i 12 AP come unità spaziali costituisce una soluzione intermedia tra l'utilizzo dei 215 comuni, dettaglio troppo fine difficilmente interpretabile soprattutto in termini di indicatori, e quello delle quattro ex Province che risulta troppo aggregato per essere informativo. Anche un eventuale riferimento alle Unioni Territoriali Intercomunali, istituite nel 2014, non avrebbe più valenza in quanto soppresse dalla LR 21/2019.

Le segnalazioni di degrado sono state classificate in 11 categorie, frutto di una rielaborazione di quelle individuate nella raccolta dei dati utili alla redazione del PPR-FVG, e sono: 1 . ambiente naturale; 2 . aree produttive e commerciali; 3 . patrimonio storico-culturale; 4. strutture e aree militari; 5 . urbano; 6 . infrastrutture viarie e di trasporto; 7. infrastrutture energetiche; 8 . cave 
AP 1 Carnia

AP 2 Val Canale-Canal del Ferro-Val Resia

AP 3 Alte valli occidentali

AP 4 Pedemontana occidentale

AP 5 Anfiteatro morenico

AP 6 Valli orientali e Collio

AP 7 Alta pianura pordenonese

AP 8 Alta pianura friulana e isontina

AP 9 Bassa pianura pordenonese

AP 10 Bassa pianura friulana e isontina

AP 11 Carso e Costiera orientale

AP 12 Laguna e Costa

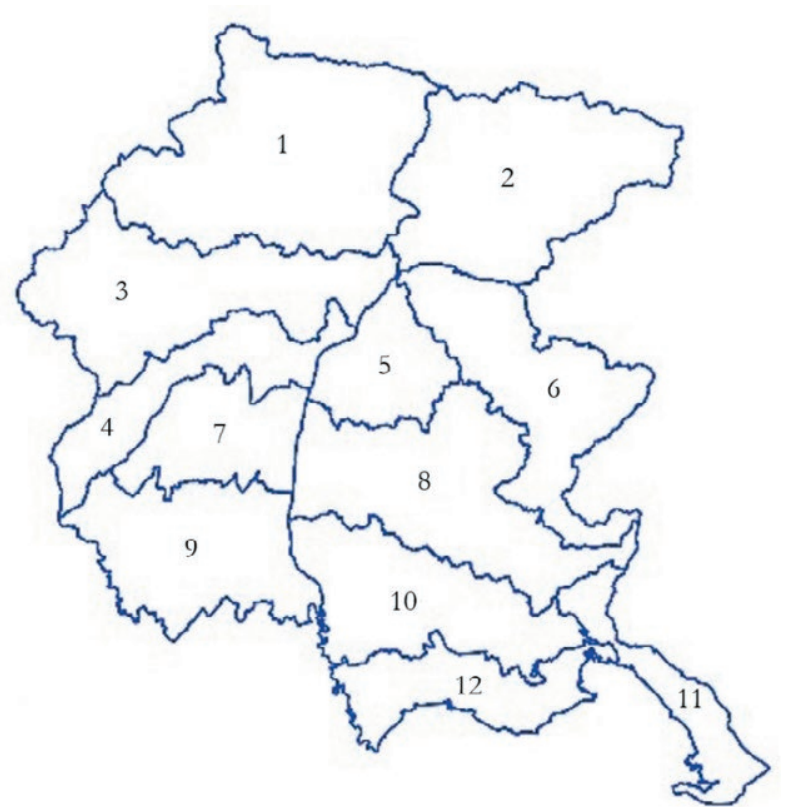

Figura 1. Perimetrazione dei 12 Ambiti di Paesaggio del FVG (Fonte: Zaccomer, 2018).

ed ex cave; 9. smaltimento rifiuti; 10 . idraulico; 11 . paesaggio rurale. Questa numerazione identifica le categorie nella Tabella 1 .

Considerato il numero di segnalazioni $s_{i j}$ nel $i$-esimo Ambito di Paesaggio per la j-esima categoria, si definisce il quoziente di Localizzazione delle Segnalazioni (LS) come un "rapporto di rapporti", ossia tra il peso di una singola categoria di segnalazione a livello di singolo AP e quello a livello regionale:

$L S_{i j}=\frac{s_{i j} / \sum_{j}^{J} s_{i j}}{\sum_{i}^{12} s_{i j} / \sum_{i}^{12} \sum_{j}^{J} s_{i j}}$

Se $L S>1$ vi è una tendenza delle segnalazioni di una particolare categoria a localizzarsi in quell'AP: può quindi essere visto come indicatore di concentrazione spaziale delle segnalazioni rispetto alla situazione regionale, ma non presenta, per costruzione, nessuna forma di normalizzazione. Questo indicatore è influenzato dal dettaglio della classificazione del degrado utilizzata (Marbach 1991), ma è possibile sintetizzare i singoli LS per uno specifico AP attraverso l'indicatore di Dissomiglianza per Ambito di Paesaggio (DAP):

$D A P_{i}=\frac{1}{2} \sum_{j}^{I}\left|\frac{s_{i j}}{\sum_{j}^{J} s_{i j}}-\frac{\sum_{i}^{12} s_{i j}}{\sum_{i}^{12} \sum_{j}^{J} s_{i j}}\right|$

Questo indicatore varia tra zero, se la composizione per AP è perfettamente identica a quella regionale, e si avvicina all'unità nel caso di massima dissimilarità. La sua lettura congiunta con i rispettivi $L S$ permette per tutti i tipi di degrado di comprendere quanto la struttura spaziale delle segnalazioni, per singolo AP, risulti difforme da quella complessiva regionale.

In base ad un primo dataset delle segnalazioni (Bressan, Pascolini 2019) - costruito per l'analisi preliminare della distribuzione spaziale delle stesse e classificate rispetto alle 11 categorie individuate - considerando solo l'indicatore $D A P$, si può constatare come le strutture per singolo AP siano alquanto diverse tra di loro. Gli AP3 e AP7 fanno registrare la massima dissimilarità, con rispettivamente 0,44 e 0,45 , ma questo è dovuto al fatto che si tratta degli AP meno coperti dalle fonti interne poiché le loro segnalazioni complessive non superano la quarantina. I valori minimi dell'indicatore sono relativi all'AP5 $(0,09)$, all'AP10 $(0,11)$ e all'AP8 $(0,15)$. Gli indicatori calcolati in Tab. 1 rappresentano il principale criterio per l'identificazione dei casi studio, anche se è utile ricordare che le segnalazioni non sono uniformi sul territorio regionale e dunque situazioni significative di eventuale degrado o abbandono potrebbero non essere state oggetto di segnalazione in quanto localizzate in aree poco rappresentate nel dataset.

Infine, si precisa che quest'analisi vuole essere un punto di partenza in quanto le segnalazioni sono poi state singolarmente trattate subendo un ulteriore processo di raffinazione e arricchimento informativo. 
Tabella 1. Indicatori calcolati per categoria di degrado. Fonte: elaborazioni degli autori (2020).

\begin{tabular}{cccccccccccccc}
\hline AP & LS1 & LS2 & LS3 & LS4 & LS5 & LS6 & LS7 & LS8 & LS9 & LS10 & LS11 & DAP \\
\hline 1 & 1,93 & 0,55 & 0,84 & 0,79 & 0,97 & 1,45 & 0,64 & 0,50 & 0,00 & 0,64 & 1,34 & 0,18 \\
2 & 1,13 & 0,45 & 0,53 & 2,19 & 0,64 & 2,23 & 0,00 & 1,09 & 0,00 & 0,00 & 2,95 & 0,31 \\
3 & 2,49 & 0,51 & 1,79 & 0,00 & 0,19 & 1,51 & 1,90 & 0,00 & 0,00 & 7,59 & 1,99 & 0,44 \\
4 & 1,59 & 0,36 & 1,08 & 0,22 & 0,89 & 1,62 & 0,72 & 4,82 & 0,00 & 2,39 & 2,25 & 0,27 \\
5 & 0,87 & 1,10 & 0,92 & 0,67 & 1,20 & 0,98 & 0,27 & 0,42 & 0,63 & 2,15 & 0,85 & 0,09 \\
6 & 0,84 & 0,45 & 1,94 & 2,01 & 1,25 & 0,60 & 0,19 & 1,18 & 1,33 & 1,14 & 1,00 & 0,22 \\
7 & 0,66 & 0,58 & 0,00 & 4,05 & 0,22 & 1,23 & 0,00 & 3,37 & 10,11 & 2,17 & 1,14 & 0,45 \\
8 & 0,46 & 1,35 & 0,61 & 1,18 & 1,12 & 0,79 & 1,54 & 0,38 & 1,32 & 0,32 & 0,68 & 0,15 \\
9 & 0,53 & 1,75 & 2,19 & 0,49 & 0,91 & 0,60 & 0,00 & 0,00 & 0,00 & 0,00 & 0,00 & 0,27 \\
10 & 0,96 & 0,97 & 1,63 & 1,07 & 0,74 & 1,08 & 2,16 & 0,00 & 2,01 & 0,00 & 1,36 & 0,11 \\
11 & 0,72 & 2,06 & 0,67 & 0,22 & 0,68 & 1,21 & 0,00 & 0,55 & 0,83 & 0,00 & 0,00 & 0,28 \\
12 & 2,04 & 0,90 & 0,39 & 0,00 & 1,27 & 0,39 & 3,13 & 0,54 & 0,00 & 0,70 & 0,37 & 0,26 \\
\hline
\end{tabular}

\section{I casi di studio}

\subsection{Il degrado delle strutture e aree militari}

La militarizzazione di una regione di confine quale il FVG è un fatto noto, ma gli aspetti dell'esatta ubicazione delle infrastrutture e della relativa occupazione del suolo sono tutt'ora poco chiari (Baccichet 2015; Corde Architetti 2016). Negli ultimi vent'anni si sono avuti diversi tentativi di quantificazione (Bianchetti 2003; Michelutti 2012), ma l'indagine conoscitiva del 2006 della IV Commissione Difesa della Camera si distingue per la sua rilevanza istituzionale (Peccol 2015). È importante notare il fatto che le informazioni, depositate dall'allora ministro Parisi, certificassero come il FVG fosse la regione italiana più interessata dalla presenza militare: il demanio militare occupava $93,1 \mathrm{~km}^{2}$, mentre le servitù militari 26,1 $\mathrm{km}^{2}$, per un totale di $1,67 \%$ della superficie regionale ${ }^{1}$.

Dalla Tab. 1 è possibile individuare gli ambiti di paesaggio con una localizzazione delle segnalazioni militari più elevata rispetto a quella regionale. Questi sono: l'ambito AP7 dell'Alta Pianura pordenonese, con $L S$ pari a 4,05 , e gli ambiti delle Alte valli orientali AP2, con 2,19, e delle Valli orientali e del Collio AP6 con 2,01. Consapevoli che la distribuzione spaziale delle segnalazioni non è omogenea sul territorio regionale, va anche sottolineato che le indagini che utilizzano i "cittadini come sensori" (Goodchild 2007), non essendo state ottenute con un piano censuario, al limite campionario, non possono essere ritenute statisticamente rappresentative della realtà. Ad esempio, nel caso dei siti militari, un unico manufatto di grandi dimensioni, come una caser-

\footnotetext{
${ }^{1}$ Cfr. www.camera.it/_dati/leg15/lavori/stencomm/04/indag/militari/2006/1025/s020.htm
}

ma abbandonata, potrebbe attirare più attenzione di decine di strutture ipogee della Guerra Fredda per loro natura ben nascoste e, probabilmente, già ricoperte da una folta vegetazione (Petruzzi, Petriccione 2019). Ecco perché non si può agire esclusivamente per via quantitativa, ma è utile esaminare ogni singola segnalazione non solo per valutarne la qualità e il contenuto informativo, ma anche per verificare se vi sono specificità che sfuggono alla mera rappresentazione numerica.

Il lavoro di revisione delle segnalazioni è stato portato a termine sfruttando le fonti esterne disponibili, validando 138 segnalazioni anche nella loro posizione geografica (Fig. 2).

Per quanto riguarda le fonti esterne utilizzate, il caso di studio delle aree militari si caratterizza per il fatto che non esistono archivi ufficiali completi (o non secretati). È quindi necessario ricorrere a ulteriori fonti esterne al progetto, che in questo caso possono essere solo costruite su base volontaria, per cui incomplete e con distribuzioni spaziali disomogenee. Per superare questi limiti è stato necessario considerarne diverse, alcune delle quali già strutturate come database spaziali: si fa riferimento all'indagine di Legambiente sulle aree militari (Coletto 2015), ai layer di OSM e alla mappa interattiva di Corde $\operatorname{Architetti}^{2}$, che per finalità operative sono stati integrati in un unico progetto la cui interfaccia grafica viene riportata in Fig. 3. Da quest'ultima si desume non solo la complessità territoriale, ma anche la scarsa uniformità delle segnalazioni militari già in evidenza in Tab. 1 .

Altre fonti esterne sono invece disponibili in forma tabellare e sono il frutto dei tentativi storici di censimento (IRDISP 1984; Democrazia Proletaria 1989), dell'inda-

\footnotetext{
${ }^{2}$ La carta interattiva di Corde Architetti è disponibile all'indirizzo http://www.primulecaserme.it/blog/?p=921
} 

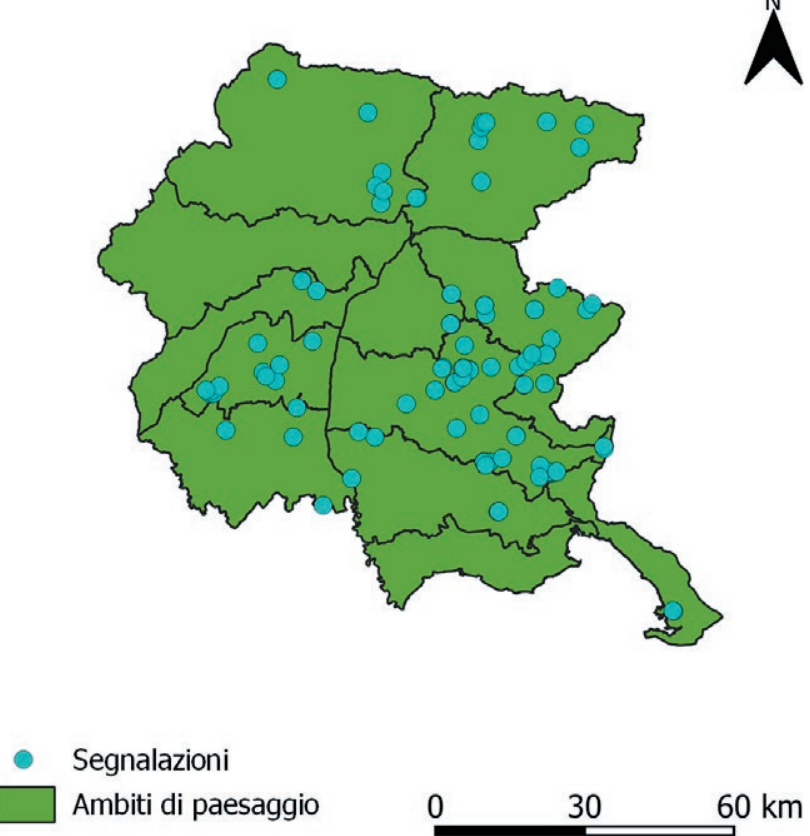

Figura 2. Segnalazioni militari regionali. Fonte: elaborazione degli autori.

gine dell'Università di Udine sulle aree dismesse (Bianchetti, Zamolo 2000; Bianchetti 2003; Bellencin Meneghel et al. 2003), e, infine, del lavoro di Corde Architetti (2016) che contiene maggiori dettagli rispetto alla già citata mappa interattiva. Sempre per verificare l'esattezza delle segnalazioni ricevute, all'evenienza sono stati utilizzati altri servizi in rete, come Google Street View o il sito dell'Agenzia del Demanio.

La revisione di ogni singola segnalazione non ha stravolto la distribuzione spaziale proposta in Tab. 1, ma ha presentato interessanti risvolti. Ecco perché si è deciso di selezionare un caso emblematico legato ai problemi qualitativi di georeferenziazione delle segnalazioni e uno invece che ha avuto pesanti ricadute socio-economiche.

Il primo caso è relativo all'ex caserma Sante Patussi che presenta una peculiare posizione geografica poiché la sua vasta area ricade a cavallo di due territori comunali: la ricognizione fisica di Tramontin et al. (2011) ha stimato circa che 5,6 ettari ricadono in comune di Tricesimo e poco più di 3 su Reana del Rojale. Questa particolarità porta a due problematiche interessanti poiché sfugge alla fotografia della Tab. 1 e, soprattutto, presenta problemi di qualità per la sua segnalazione geografica. Infatti, se Tricesimo appartiene all'AP5 dell'Anfiteatro morenico, parte di Reana invece appartiene all'AP8 dell'Alta pianura friulana e isontina: il territorio della Sante Patussi insiste su due AP diversi e, quindi, le sue segnalazioni vengono ripartite su questi ambiti. Per questo le segnalazioni devono essere precise altrimenti anche poche decine di metri spostano il riferimento da un ambito all'altro: durante l'operazione di validazione è emerso come per alcune segnalazioni, pur collocando

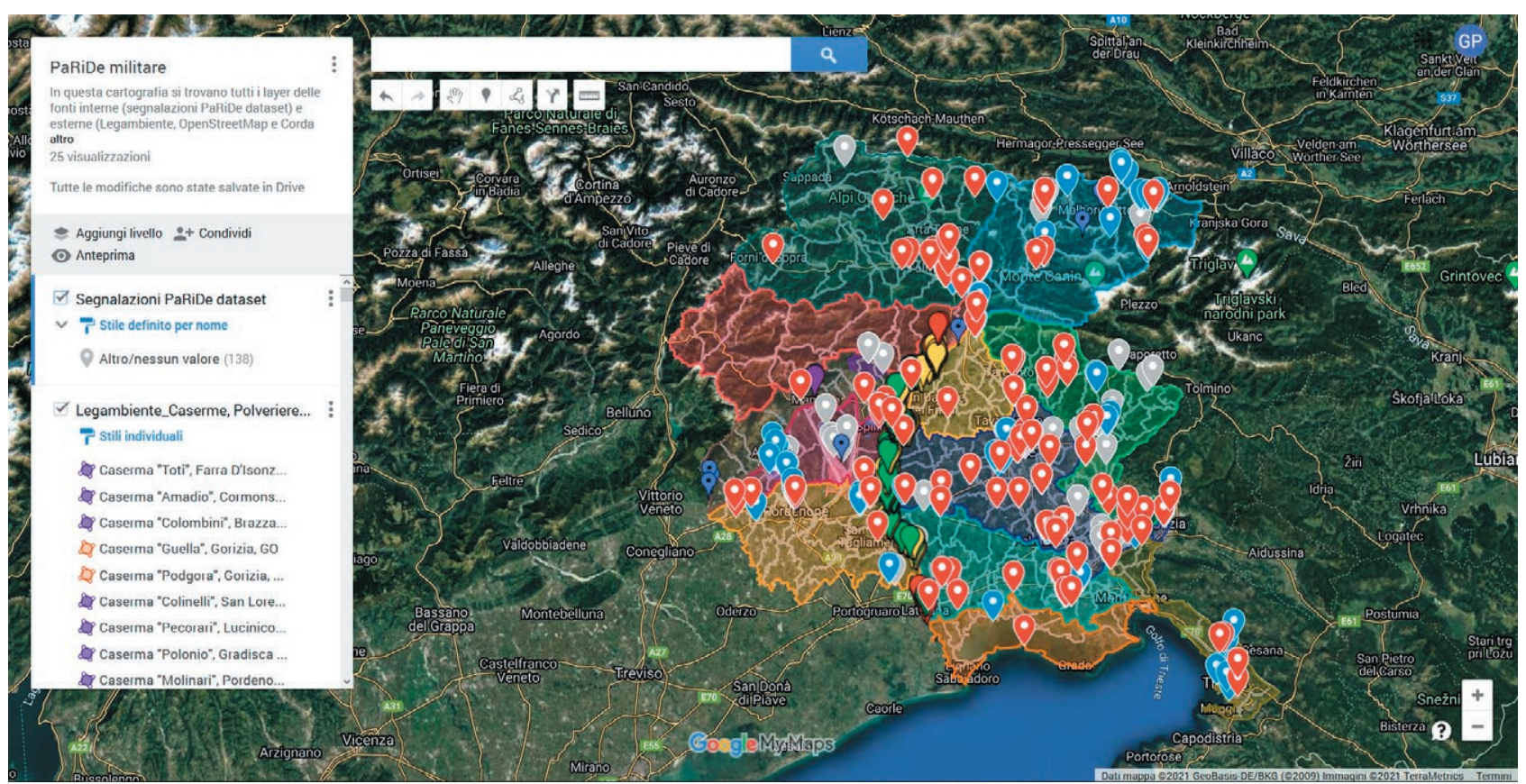

Figura 3. Interfaccia grafica dei database militari. Fonte: elaborazione degli autori, basata su Google Maps su fonti interne ed esterne. 

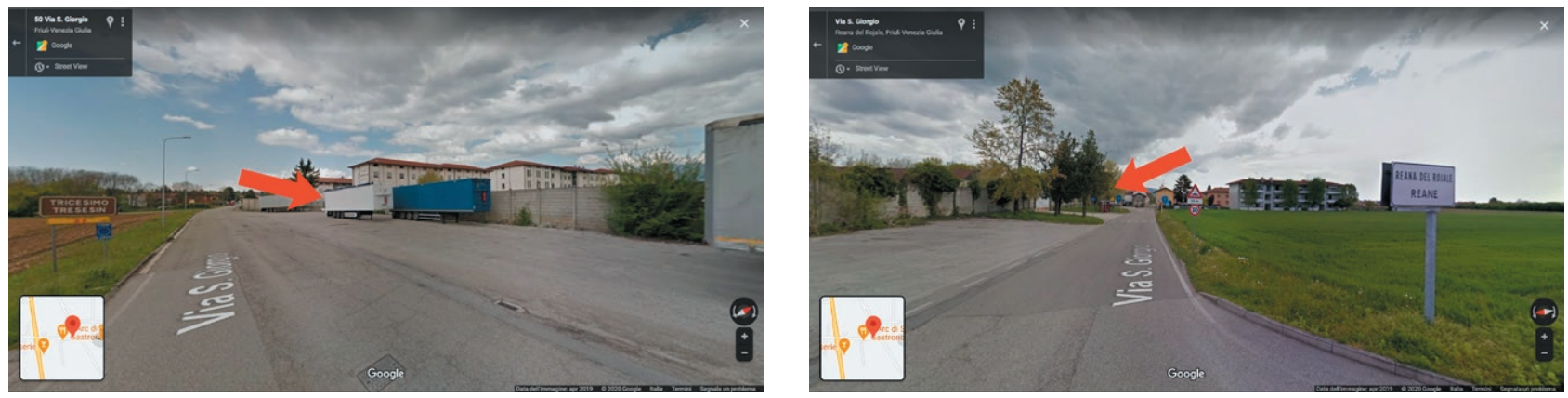

Figura 4. Entrata della caserma da Tricesimo (a sinistra) e ingresso su Reana del Rojale (a destra). Fonte: Google Street View (immagini di aprile 2019).

testualmente la caserma in un comune, la georeferenziazione fosse riferita al comune contiguo. In realtà, il problema non è solo legato alla percezione della sua ubicazione, ma anche in ragione del fatto che lungo la strada che costeggia la caserma è posizionato il cartello del cambio di competenza comunale esattamente tra i due ingressi all'area (Fig. 4). Le collocazioni non coerenti con la segnalazione testuale sono state ripristinate e, quindi, il layer del dataset PaRiDe militare è stato opportunamente integrato e corretto.

La storia della dismissione e del recupero della Sante Patussi viene proposto da Baccichet (2015) come esempio di difficoltoso intervento di recupero. Infatti, una volta che le aree militari vengono ufficialmente dismesse e cedute, i comuni interessati si trovano spesso impossibilitati a procedere con il recupero tenuto conto che, come nel caso della Sante Patussi, le aree coinvolte sono spesso ampie: "Mancando idee e danaro per investimenti a volte la conclusione più immediata è quella di cedere ai privati il bene ereditato da Stato e Regione. Ma anche la scelta di fare cassa non è sempre facile [...] La Patussi a Reana del Rojale è andata all'asta, ma nessuno ha presentato un'offerta" (Baccichet 2015, 62). A tal proposito, è stata chiamata in causa anche l'Università di Udine per la costruzione di un processo partecipativo che tenesse conto delle aspettative dei principali stakeholder (Tramontin et al. 2011), ma non mancano altre proposte di recupero $^{3}$. Dal 2018 parte della caserma ospita un museo militare gestito da volontari, ma l'azione di recupero dei restanti edifici rimane un nodo irrisolto come testimoniato dalle numerose segnalazioni raccolte da questo progetto che evidenziano come l'area sia percepita come ancora bisognosa di riqualificazione.

Il secondo caso è stato invece selezionato per il forte impatto socio-economico sulla comunità di Pontebba,

${ }^{3} \mathrm{Cfr}$. http://www.laboratoriodipaesaggifvg.it/progetto-di-riqualificazione-dellex-caserma-patussi-tricesimo-studio-di-fattibilita/ località che si trova nell'asse vallivo tracciato dal Fiume Fella, nel Canal del Ferro. Ponendo l'attenzione sulle dismissioni, non solo militari, presenti in questo territorio, Guaran (2003, 334-335) scrive che "parecchi beni e aree in dismissione o già dismesse [sono] da mettere in stretta relazione con l'importante funzione per il trasporto rivestita dal corridoio del Fella [...]. Le proprietà degli stessi risulta, per i più, del demanio dello Stato e, per un numero più contenuto delle Ferrovie dello Stato, degli Enti locali o di privati". Nel caso di Pontebba, le caserme sono posizionate poco distanti dalla ferrovia dove è presente un grande scalo, anch'esso ormai in disuso (Fig. 5). Le segnalazioni ricevute hanno evidenziato l'ex caserma della Guardia di finanza Gollino

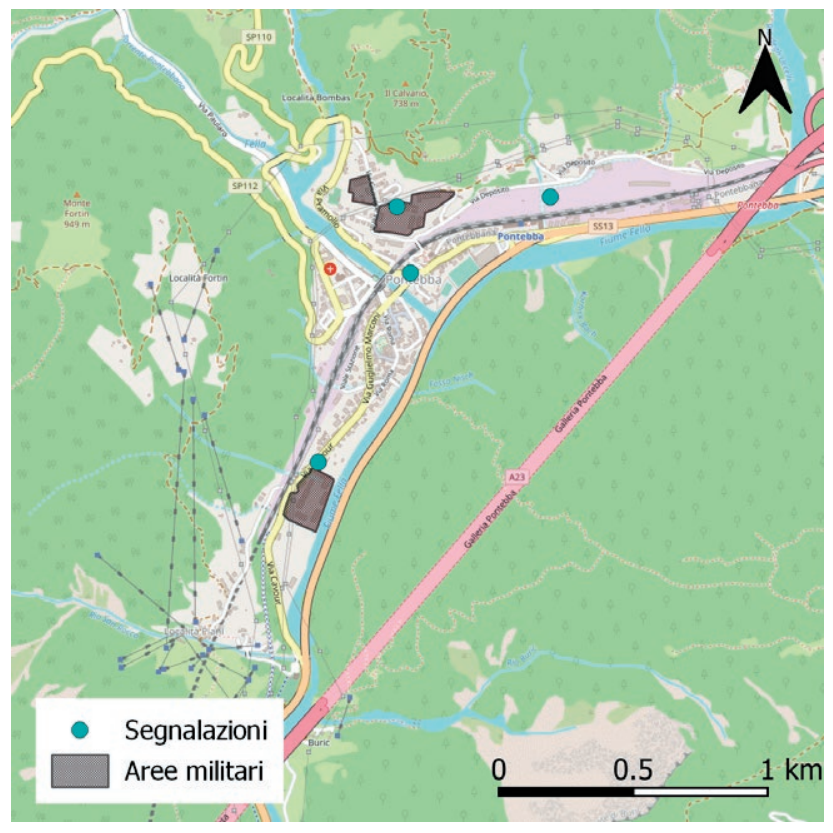

Figura 5. Aree (poligoni OSM) e segnalazioni militari a Pontebba. Fonte: elaborazione degli autori (c) OpenStreetMap contributors. 
e Marinelli, e le due ex caserme dell'esercito Bertolotti e Zanibon, nonché la vasta area dello scalo.

In riferimento a questo comune montano, viene segnalata l'importanza non solo dei beni dismessi militari e legati alla ferrovia, ma anche delle aree gestite dalla Guardia di Finanza che servivano per l'espletazione della funzione doganale prima dell'entrata dell'Austria nell'Unione Europea (1995). Baccichet (2015) illustra come l'intera struttura socio-economica di Pontebba, compresa quella demografica, ruotasse attorno ai militari, ai finanzieri e ai dipendenti delle Ferrovie Italiane: l'emigrazione di queste tipologie di lavoratori non poteva che far ricadere il paese nelle problematiche delle aree montane del FVG, lasciando però in eredità anche il problema degli impatti relativi alle strutture abbandonate. Infatti, la presenza di più ex caserme militari e della Guardia di Finanza è andata a gravare sull'amministrazione comunale alle prese con una situazione demografica critica - ad inizio anno 2021 l'ISTAT dichiara 1.353 residenti, soprattutto anziani, a fronte delle 2.503 persone nel 1981 - fornendo un ulteriore esempio della "retorica del dono" statale, nonostante per il caso Pontebba non siano mancate le ipotesi di recupero e l'amministrazione comunale abbia stanziato dei fondi (Corde Architetti 2016, 224-225).

In base all'analisi condotta sulle segnalazioni relative al degrado delle strutture e delle aree militari appare evidente la necessità di un archivio esaustivo ufficiale, poiché il lavoro di analisi delle segnalazioni risulterebbe più semplice e di maggiore qualità se l'amministrazione regionale decidesse di censire definitivamente tutte le aree e le infrastrutture militari, stimate più del doppio di quelle qui analizzate (Corde Architetti 2016), e di rendere pubblico l'archivio almeno per quelle non più coperte da segreto militare, favorendo così un iter di recupero o riconversione di queste aree.

\subsection{Abbandono e dissesto idraulico fluviale}

Il complesso reticolo idrografico del FVG è uno degli aspetti geografici più rilevanti per studiare, analizzare e comprendere il territorio regionale (De Cillia 2000). La storica necessità delle comunità antropiche di venire a patti con l'idrografia superficiale ha dato vita a dei processi di territorialità anfibia i cui esiti possono essere apprezzati leggendo gli strati e le sedimentazioni di specifici paesaggi ibridi che riflettono la "naturaleartificiosità" dei corsi d'acqua (Lefebvre 1991; Smith 1984). Il costante e continuo interfacciarsi tra gli attori territoriali e il supporto ambientale ha favorito, da un lato, lo sviluppo delle attività materiali, nonché di speci- fici assetti geo-culturali e identitari, dall'altro, ha portato con sé conflitti originando paesaggi contesi (Swyngedouw 2015; Vallerani 2004). La gestione, la manutenzione e i continui interventi per garantire il delicato equilibrio che sottende il controllo delle acque, la loro percezione e l'utilizzo in negativo e/o positivo, sono un tema rilevante e per questo è stato scelto tra i possibili ambiti di indagine delle segnalazioni come categoria meritoria di approfondimento (Cosgrove, Petts 1990; Minoia, Faggi 1995; Vallerani, Visentin 2018).

In relazione alle fonti interne sono stati utilizzati i risultati ottenuti tramite il progetto PaRiDe e il webGIS. Da questi dati sono state selezionate le segnalazioni di dissesto/abbandono/degrado afferenti alla categoria 10 (Degrado idraulico) alla quale sono state associate, per ottenere una casistica più estesa, le segnalazioni della categoria 1 (Degrado in ambiente naturale) ritenute affini o che avessero una rilevanza per gli ambiti fluviali, lacuali e lagunari. Questo ha permesso di ampliare l'indagine a 145 segnalazioni, ripartite per bacini idrografici grazie all'elaborazione di uno specifico layer cartografico, ritenuto più congeniale per lo studio (Fig. 6).

Nel tentativo di far dialogare le informazioni comunicate dai cittadini con il sapere esperto/istituzionale (fonte esterna), è stata utilizzata relativamente agli eventi

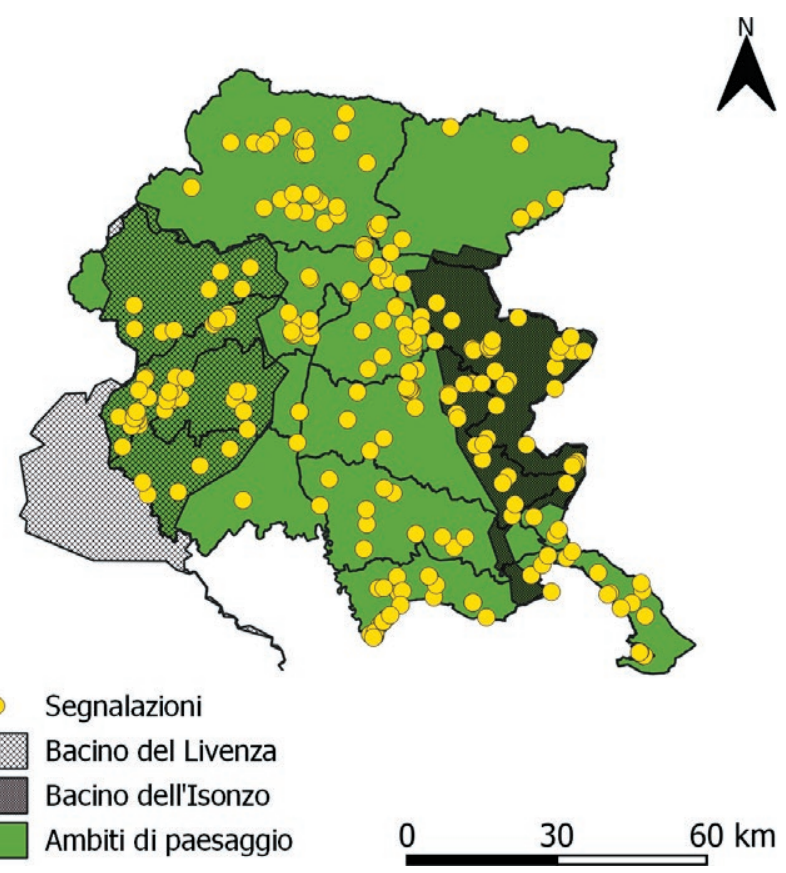

Figura 6. Carta della distribuzione delle segnalazioni della categoria idrico/idraulico con in evidenza la sovrapposizione tra gli ambiti di paesaggio e i bacini idrografici. Fonte: elaborazione degli autori. 
di dissesto idrogeologico la banca dati regionale IRDAT ${ }^{4}$ che ricomprende un totale di 1550 elementi, avendo escluso tutti i record non accompagnati da informazioni qualitative e descrittive.

Per integrare le fonti esterne e così contestualizzare le segnalazioni (fonti interne), si è ricorsi alle schede cartografiche PAI (Piani stralcio per l'assetto idrogeologico), a cura dell'autorità di Bacino, che indicano il grado di pericolosità idraulica su quattro livelli (moderata, media, alta e molto alta) relativamente ai bacini del Tagliamento, dell'Isonzo e della Livenza. Mentre per i tributari della Laguna di Marano e Grado, del torrente Slizza e del Levante i dati provengono dal PAIR (Piano stralcio per l'assetto idrogeologico dei bacini di interesse regionale).

E doveroso ricordare che le segnalazioni provenienti dalle fonti interne avevano l'obiettivo di raccogliere situazioni ritenute rilevanti da parte della cittadinanza all'interno di una cornice di riferimento relativa ai paesaggi del valore e del degrado. Diversamente, le banche dati scelte per il raffronto (IRDAT, PAI e PAIR) sono legate a specifiche situazioni di dissesto idrogeologico e/o di rischio esondazione, e raccolgono dati elaborati da tecnici regionali o appartenenti ad enti di gestione dei corpi idrici (es. Consorzi di bonifica) con lo specifico intento di mappare lo stato dell'arte in relazione a interventi da effettuare o realizzati, in genere di matrice tecnico-ingegneristica. Questo raffronto ha indotto a riflettere sulla provenienza e sulla qualità del dato. I linguaggi, gli obiettivi e le sensibilità espressi dai diversi attori territoriali permettono di iniziare a ragionare sul rapporto tra "sapere esperto" e "sapere contestuale/ cittadino".

I casi studio sono stati selezionati prendendo in considerazione il quoziente di localizzazione delle segnalazioni per la categoria idraulica (LS10) e quella del degrado in ambiente naturale (LS1). Infatti, nonostante non vi sia una esatta sovrapposizione tra bacini idrografici e ambiti di paesaggio, in quanto i presupposti per le zonizzazioni sono concettualmente differenti, l'incrocio degli indicatori di localizzazione con la cartografia relativa agli AP (intersecando i bacini idrografici) ha permesso di identificare alcuni tratti fluviali particolarmente significativi in ragione del valore medio-alto dei quozienti localizzativi calcolati. Per esempio, per quanto riguarda il bacino del Livenza, in corrispondenza degli AP 3 e 4, i rispettivi quozienti sono 7,59 e 2,39, i più alti della categoria come nel caso del bacino dell'Isonzo il cui quoziente è di 2,15 (AP5); analogamente nei medesi-

\footnotetext{
${ }^{4}$ IRDAT è l'Infrastruttura regionale dei dati ambientali e territoriali. Il portale è accessibile all'indirizzo: http://www.regione.fvg.it/rafvg/cms/ RAFVG/ambiente-territorio/conoscere-ambiente-territorio/FOGLIA2/
}

mi AP risultano tra i più alti i valori dell'indicatore LS1.

Per il bacino del Livenza sono state considerate le segnalazioni in comune di Tramonti di Sopra, a sud del centro del paese, poco prima della confluenza nel Meduna del torrente Viellia. Nell'archivio partecipato, un segnalatore nel 2016, dopo aver valutato il sito con un punteggio molto basso (1 sia nel suo complesso che a livello naturalistico/ambientale ed estetico/evocativo) ${ }^{5}$ descrive il sito come “...da decenni la 'spiaggia' del villaggio. Qui sono stati costruiti i servizi per la sosta e si sono attrezzate le aree per il picnic. Recentemente la Regione ha operato una radicale ricalibratura dell'alveo facendo scomparire le ampie pozze e le rive dolci di ghiaia. Sistemando poi delle traverse e dando al luogo un aspetto del tutto nuovo e mal digerito dalla popolazione locale”. Da questa descrizione emerge un certo disappunto per gli interventi che sono stati approntati per la gestione dell'asta fluviale, confermati dalla banca dati IRDAT (nel 2004 e nel 2009) che sottolinea diversi problemi relativi all'erosione delle sponde, sia lungo il Meduna, che all'altezza del torrente Viellia a monte del centro di Tramonti di Sopra, nonché leggermente più a valle, in corrispondenza della località Tramonti di Mezzo. Il medesimo segnalatore specifica: "I cittadini sono arrabbiati per la semplicistica lettura che è stata data di questo ambiente senza considerare che le opere hanno modificato radicalmente l'aspetto dei luoghi con la giustificazione della stabilità che in realtà era garantita da secoli e secoli. Mai il fiume aveva eroso come in questi anni recenti a seguito della sistemazione idraulica". In questo caso sembra che non vi sia consonanza tra le opinioni di alcuni cittadini (nell'archivio sono presenti altre segnalazioni dello stesso tono) e il sapere esperto che chiamato in causa per la messa in sicurezza idraulica (forse su segnalazione dei proprietari o dell'ente comunale?) si occupa di prevenire il rischio con soluzioni ingegneristiche puntuali senza valutare con attenzione il contesto storico-culturale, le aspettative e le pratiche dei residenti.

Un secondo caso, sempre afferente al bacino del Livenza, riguarda i torrenti Midella e Ossena in comune di Aviano, a nord del centro abitato. Qui scorre il torrente Midella, esondato nel 2011, il quale si unisce al torrente Ossena poco prima di entrare nel centro urbano. I due corsi d'acqua, dopo la confluenza, costeggiano il centro abitato, in un letto canalizzato con sponde di massi, frutto di un intervento di regimazione per evitare esondazioni, ma che con molta probabilità crea un aumento

\footnotetext{
${ }^{5} \mathrm{Si}$ precisa che nel webGIS oltre a una valutazione complessiva, attribuendo un punteggio da 1 (alta criticità) a 6 (alto valore), era possibile esprimere, ricorrendo alla medesima modalità, anche giudizi specifici su singoli livelli di significato.
} 
della velocità dei deflussi a causa della sezione e della forma rettilinea conferita al corso d'acqua.

Un segnalatore nel 2016 attribuisce al sito un punteggio medio (3) riconoscendo la logica delle azioni di prevenzione, ma non la successiva manutenzione e gestione del corso e delle opere. Nel descrivere il corso afferma che: "Il Midella è un torrente di Aviano che per molti periodi dell'anno risulta in secca e che nei periodi di forte pioggia riesce a riempirsi comportando, soprattutto fino a qualche anno fa, problemi di allagamento in prossimità delle sponde. Alcuni lavori di sistemazione eseguiti di recente hanno consentito di ovviare a molte di queste problematiche; allo stato attuale però il torrente si trova in pessime condizioni di manutenzione e il bosco si sta appropriando di molti tratti del suo percorso".

Quanto riportato dal segnalatore trova una conferma nei dati IRDAT in quanto 8 tratti sono stati individuati come aree di rischio in relazione ad erosione spondale o esondazione e oggetto di interventi, realizzati per la tutela dei territori circostanti e per la prevenzione, con l'obiettivo di attenuare gli effetti in caso di possibile esondazione. Queste modifiche hanno determinato e aumentato l'artificializzazione del corso e ridotto l'accessibilità alle sponde. I vincoli di tutela e gli interventi idraulici vengono più che altro percepiti come privazioni e/o impedimenti, forse perché nell'ottica di una valorizzazione attiva si dovrebbe prevedere o garantire non solo la sicurezza ma anche l'accessibilità. Infatti, il distanziamento dal "bene fiume" riduce le opportunità per i cittadini di relazionarsi quotidianamente col corso d'acqua, come sottolineato da un segnalatore sempre nell'area di Aviano: "impediscono ai privati e alle associazioni che fino a qualche anno fa si occupavano della pulizia e della manutenzione dei torrenti del territorio di intervenire". Si riporta a tal riguardo una proposta che in realtà si avvicina ad un modello di gestione partecipata del territorio: "Potrebbero essere alleggeriti i vincoli che impediscono ai privati di prendersi cura del territorio. Molti sono giusti e corretti, ma potrebbero essere tutelate e incentivate anche le attività che vanno a vantaggio del territorio e non a suo discapito. Alcuni enti del comune avianese organizzavano fino a qualche anno fa, grazie al lavoro di volontari, la pulizia e la manutenzione dell'alveo".

Relativamente al bacino dell'Isonzo, nello specifico in riferimento al torrente Torre, si sofferma l'attenzione su due rii, Lavandar e Indrella, in comune di Tarcento, tra le località di Villafredda e di Segnacco. La segnalazione (2016), inserita nella categoria naturalistico-ambientale e con una criticità bassa (punteggio 3), recita: "Rii drenanti la porzione meridionale del territorio di Segnacco, pesantemente condizionati nel loro percorso dalle vecchie bonifiche, che hanno eliminato le antiche pozze ed anse naturali, ma oggi parzialmente rinaturalizzati in concomitanza delle rive sebbene solo per pochi metri a causa della pressione dei campi agricoli circostanti", mettendo in luce lo storico conflitto tra la volontà di tutelare i beni naturali (corsi d'acqua e vegetazioni ripariali) e le esigenze produttive dell'economia agricola. Di interesse, anche se riferita a una sezione di uno dei due corsi d'acqua posto poco più a valle, la fonte regionale IRDAT (2001). Questa sottolinea la presenza di fenomeni erosivi spondali in un tratto del Torre caratterizzato da una pericolosità idraulica elevata (PAI). La domanda implicita riguarda le bonifiche e le sistemazioni idrauliche: a giudizio del cittadino avevano influenzato negativamente lo scorrere dell'acqua e probabilmente il tracciato stesso del rio. Sembra che queste operazioni abbiano potuto determinare in qualche modo l'azione erosiva e i conseguenti danni evidenziati dalla fonte regionale.

\subsection{I siti di cava}

Le segnalazioni raccolte tramite il progetto evidenziano un'attenzione della cittadinanza anche per quelle situazioni in cui la compromissione del paesaggio deriva dalla prevalenza delle finalità economiche sugli obiettivi legati alla conservazione del patrimonio naturale. Infatti, l'attività estrattiva, soprattutto quella condotta a cielo aperto, incidendo direttamente nella componente geomorfologica del terreno, crea un "visibile-evidente" impatto sul paesaggio (Gisotti 2008, 109).

In questo progetto, $\mathrm{i}$ dati percettivi legati alle cave sono stati confrontati sia con un dataset geografico di fonte IRDAT, sia con le geometrie estraibili da OSM. Nel primo caso, la geometria di ogni elemento corrisponde al perimetro autorizzato delle cave che hanno un decreto vigente al 12 dicembre $2019^{6}$. Per quanto riguarda la fonte OSM, l'estrazione, effettuata nel luglio 2020, ha dato luogo alla creazione di un layer con 194 elementi areali. Di questi 98 non hanno una denominazione ma solo una rappresentazione cartografica. Dalla tabella degli attributi si può dedurre che 69 elementi non sono opera di mappatura volontaria, ma provengono da altre fonti, inclusa l'IRDAT. Sono solo 29 gli elementi nel dataset OSM che sono allo stesso tempo originali, perché non presenti in altre fonti, e corredati da qualche informazione qualitativa. Dei poligoni di OSM, 16 elementi vengono chiamati esplicitamente "ex-cave".

Passando alle fonti di dati non georeferenziati sulle ex-cave è necessario fare riferimento ad un documento

${ }^{6}$ Per maggiori informazioni sui metadati: http://irdat.regione.fvg.it/consultatore-dati-ambientali-territoriali/detail/irdat/dataset/1556 


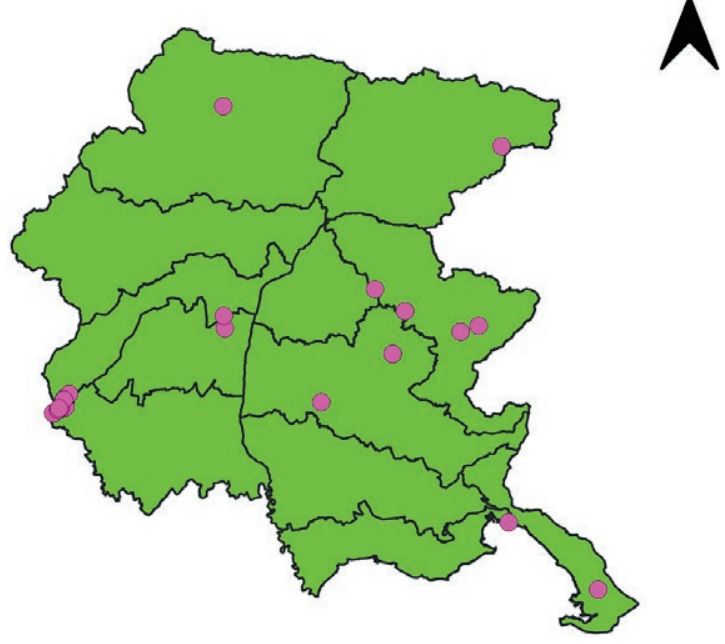

\section{- Segnalazioni}

Ambiti di paesaggio

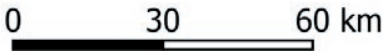

Figura 7. Segnalazioni riguardanti cave ed ex cave. Fonte: elaborazione degli autori.

regionale (Regione Autonoma FVG 2018b) in cui vengono elencate sette aree di cave dismesse ${ }^{7}$, distribuite in sei comuni della regione. È interessante notare che mentre è palese che in OSM si è avuta l'importazione di alcune geometrie dal dataset di cave attive di IRDAT (nel campo "descriptio" ci sono 69 elementi che hanno come fonte un url della regione), gli utenti di OSM non hanno fatto uso delle informazioni presenti in questo documento regionale per popolare ulteriormente il dataset geografico.

La Figura 7 individua la localizzazione delle segnalazioni dei siti di escavazione, in totale 27, suddivisi per ambito di paesaggio. La pedemontana occidentale (AP4) è l'ambito a cui è associato, per la categoria in questione (LS8), un valore dell'indicatore di localizzazione maggiore $(4,82)$. Qui si trova infatti il comune di Caneva, dove si incontra il maggior numero di segnalazioni (13). Lo stesso comune risulta in regione anche quello con il numero più elevato di cave attive, sei, secondo il dataset IRDAT. Per sei segnalazioni, ricadenti nei comuni di Remanzacco, Basiliano, Cividale, Reana del Rojale e

\footnotetext{
${ }^{7}$ L'articolo 3, comma 1, lettera b) della legge regionale 12/2016 sulla disciplina delle attività estrattive (Regione Autonoma FVG 2016) definisce larea di cava dismessa come "la porzione del territorio interessata da una pregressa attività estrattiva in cui, in assenza di garanzia fideiussoria, non è stato effettuato il riassetto ambientale dei luoghi". E dunque necessario essere cauti quando si compiono confronti fra queste cave definite dalla legge e le percezioni dei cittadini su questa tema, dal momento che la definizione regionale è piuttosto ristretta e tecnica.
}

Tarvisio, si constata che il database IRDAT è insufficiente per l'analisi, in quanto in queste località non risulta la presenza di cave con decreto vigente associato. Tale osservazione sottolinea dunque la necessità di integrare il dataset ufficiale con altre fonti dal momento che la percezione di degrado può essere scaturita anche dalla presenza di cave inattive. Utilizzando il dataset OSM per ulteriori confronti, risulta che sia a Remanzacco, Reana del Rojale e Tarvisio ci sono degli elementi classificati come cave, giustificando dunque le segnalazioni dei cittadini. In corrispondenza di Cividale e Basiliano, OSM non presenta geometrie. Tale risultato deve essere letto con attenzione. In prima istanza OSM essendo un database volontario può non essere aggiornato, preciso ed esaustivo. Non si può escludere però che la presenza di una segnalazione in un comune privo di cave o ex-cave sia il risultato di un errore di attribuzione o interpretativo. Infatti, lo scarso contenuto informativo di talune segnalazioni incide sulla possibilità di costruire un database geografico accurato (Bressan, Pascolini 2019).

I dati percettivi raccolti consentono di effettuare ulteriori osservazioni di natura qualitativa e a tal proposito si vuole fornire qualche esempio. Le segnalazioni afferenti a questa tematica raccolte tramite il webGIS sono la maggioranza (15), ma non consentono di effettuare approfondimenti, in quanto i testi si limitano principalmente a descriverne la localizzazione (e.g. "Cava Filaferro visibile dal ponte sul Torre"). Per quanto riguarda l'altra fonte, il progetto PaRiDe, il geo-questionario (Bressan, Amaduzzi 2020) prevedeva per ogni segnalazione non solo una mappa online in cui indentificarne la posizione ma anche una domanda aperta sulle ragioni del possibile degrado. Una segnalazione di "ex-cava" raccolta con questa modalità è posizionata su Basiliano, comune in cui, secondo i database georeferenziati selezionati (IRDAT, in cui sono presenti solo cave attive; OSM, in cui ci sono sia cave ed ex-cave) non sono presenti siti di escavazione. A Basiliano vi è una ex-cava, secondo il decreto regionale concernente l'individuazione delle aree dismesse (Regione Autonoma FVG 2018b). Di questa segnalazione è inoltre interessante esplorare l'aspetto qualitativo, dal momento che nella domanda sulle ragioni di degrado la risposta è "presenza di rifiuti". Questo esempio evidenzia in primo luogo che il database OSM non censisce tutte le ferite paesaggistiche ascrivibili alle cave, in ragione della difficoltà per un utente di OSM, che non conosce direttamente la località, di individuare e classificare questo sito come ex-cava, in particolare se parzialmente ricoperto da vegetazione (Fig. 8). Nel caso delle cave dismesse, dal momento che i 'vecchi' perimetri di cava autorizzati non hanno alcun valore ai fini della disci- 


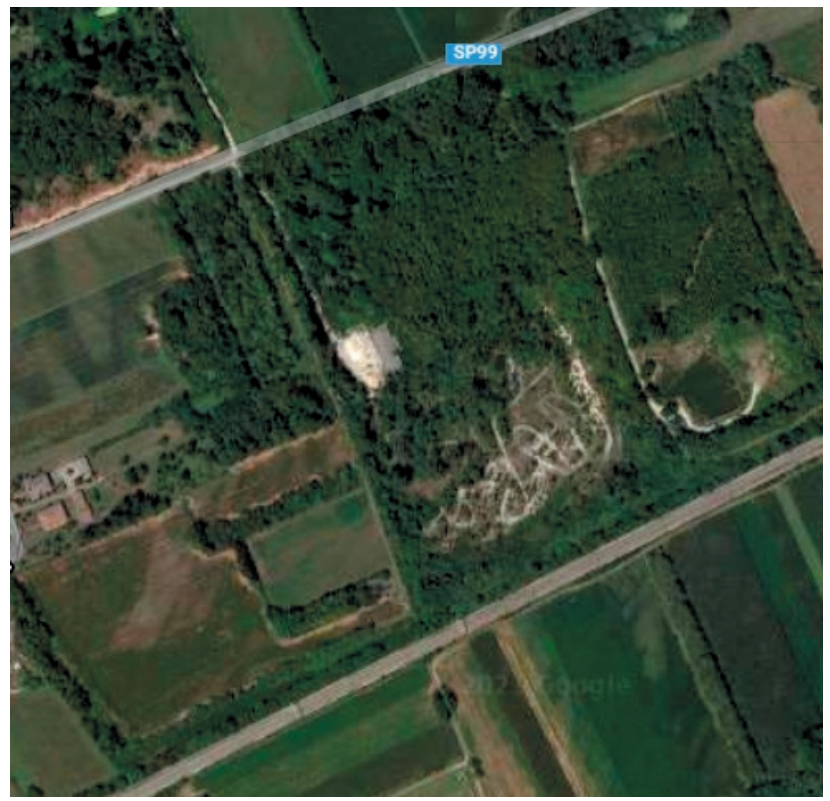

Figura 8. Cava dismessa a Basiliano. Fonte: Google Maps (immagini settembre 2021).

plina regionale delle attività estrattive (Regione Autonoma FVG, 2016), è comprensibile che l'esistenza di un eventuale dataset a elementi poligonali di natura istituzionale sulle ex-cave avrebbe solo finalità conoscitive e non legali. Questa segnalazione fa emergere anche il problema del recupero ambientale delle cave dismesse. A una dismissione non seguono sempre, come auspicato, interventi di rivalorizzazione ambientale o interventi di mitigazione, ma in realtà "[m]olte di queste aree versano in uno totale stato di abbandono, adibite allo stoccaggio indiscriminato di materiale di ogni genere" (Pieri Buti 2007, 26).

Un'altra segnalazione nel comune di Trieste indica "cave dismesse nel Carso", e come spiegazione fa riferimento all'"inquinamento". Anche in questo caso si allude a cave dismesse. In OSM ci sono di fatto degli elementi geometrici ascrivibili a cave nel comune di Trieste, però non è possibile ricondurre la segnalazione ad una cavità precisa a causa della scarsa completezza del database esterno di natura volontaria. Inoltre, il contributo del partecipante è solo parzialmente di aiuto nel creare nuova conoscenza in quanto la scelta è stata quella di utilizzare una geometria puntuale per segnalare un congiunto di oggetti, creando dunque qualche difficoltà al ricercatore che non è nelle condizioni, senza ulteriori elementi descrittivi, di identificarlo puntualmente. Il decreto regionale con l'elenco delle ex cave non evidenza la presenza di questi elementi nel comune di Trieste. Esistono però, secondo questa fonte, due cave dismes- se nel vicino comune di Monrupino che evidenziano la possibilità che il partecipante abbia commesso un errore nell'esercizio di mappatura, posizionando la segnalazione in un comune sbagliato.

A conclusione di questi confronti è significativo osservare come questi siti di notevole superficie riscontrino poco interesse sia per il cittadino, nella sua veste di sensore, che per il mapper di OSM. Da un lato, la scarsa numerosità di segnalazioni potrebbe testimoniare come queste ferite nel territorio siano diventate in realtà elementi paesaggistici della quotidianità, non più notati per il loro impatto. Dall'altro, il fatto che nel progetto di cartografia collaborativa ci siano dei problemi associati a queste geometrie (assenza di informazione correlata ai poligoni) sottolinea come probabilmente ci sia una certa difficoltà nell'attribuire un'etichetta e forma certa ad ampie superfici areali caratterizzate per avere confini mobili (cave) o per essere aree, per lo più inutilizzate, in terreni privati (ex cave).

\section{Conclusioni}

Uno degli obiettivi principali del presente contributo era quello di indagare le possibili connessioni tra il sapere esperto e quello contestuale ai fini di comprendere le potenzialità e le difficoltà nel mettere in relazione le diverse conoscenze per favorire la gestione del territorio. Per conseguire questo obiettivo si è fatto riferimento, in chiave esemplificativa, a tre categorie di criticità paesaggistiche emerse in occasione del percorso di coinvolgimento attuato nel quadro progettuale PaRiDe che a sua volta aveva conglobato i dati sulla percezione dei cittadini raccolti durante l'elaborazione del Piano Paesaggistico Regionale (Regione Autonoma FVG 2018a). L'eterogeneità dei dati di partenza ha confermato come ogni tematica, e area geografica, siano sensibili a sollecitazioni e risposte specifiche, comportando risultati diversi in base alla scelta che viene operata quando si confrontano saperi esperti e contestuali, e/o database esterni ed interni.

Il presente lavoro, da un lato, fornisce delle indicazioni di carattere metodologico a chi volesse affrontare simili analisi in altri contesti e per eventuali percorsi di approfondimento al fine di far dialogare i diversi saperi territoriali (esperto/contestuale) con l'obiettivo di conseguire una loro più efficace interazione che vada oltre la racconta dei dati o la consultazione dei documenti. Dall'altro, conferma come l'esperienza del cittadino possa portare alla luce informazioni non note e, quindi, possa permettere di arricchire il patrimonio informativo, a patto che quest'ultima esca da una logica episodica per confluire in un sistema consolidato. 
Sul piano operativo, si vuole porre l'attenzione su tre livelli, che rappresentano questioni sensibili che potrebbero essere affrontate in future esperienze di cartografia partecipativa.

In primo luogo, i dati raccolti dall'attività progettuale offrono solo in parte garanzie in termini di qualità, in particolare in relazione alla precisione nel posizionamento della segnalazione e alla completezza dell'informazione pervenuta come ben esemplificato nel caso delle strutture e aree militari. Questi problemi si riscontrano in molti progetti di cartografia partecipativa e, in parte, si possono correggere attivando forme forti di coordinamento fra proponente e partecipante.

L'altra questione rilevante riguarda la rappresentatività e la copertura spaziale delle segnalazioni. Relativamente all'aspetto statistico, i partecipanti non sono stati selezionati tramite metodi campionari ed è stata lasciata ampia discrezionalità nell'identificazione di una situazione di degrado. Queste decisioni influiscono sulla capacità di raccogliere segnalazioni, favorendo una certa difformità spaziale e percettiva. Proprio per capire gli effetti della difformità spaziale, risulta sempre consigliabile costruire delle statistiche descrittive delle segnalazioni così come è stato proposto in questo lavoro con l'elaborazione del quoziente di localizzazione (LS) e l'indicatore di dissomiglianza (DAP). Pur con la consapevolezza di queste problematiche, si vuole sottolineare che si è fatto largo uso della rete per raggiungere i potenziali partecipanti così pure si è cercato di coinvolgere persone con diverse sensibilità, indirizzando le varie indagini verso categorie specifiche.

Infine, a proposito delle fonti esterne, si sottolinea che la loro selezione risulta parziale non essendo sempre garantita la loro notorietà, unicità e ufficialità. Soprattutto nel caso di dataset esterni partecipativi, possono sorgere dunque dubbi sulla precisione e attendibilità delle fonti, facendo emergere la necessità di procedere con una valutazione critica dell'informazione esistente. OSM, il progetto di cartografia partecipativa più conosciuto al mondo, raggruppa dati georeferenziati creati da un'ampia comunità di soggetti, dove però l'inserimento dell'informazione risponde (in principio) a precise linee guida e per il quale esiste una procedura di validazione del dato. Dunque, se da un lato esiste una forma di controllo di qualità del dato, non presente ad esempio nel momento dell'inserimento dei dati del webGIS del progetto friulano, dall'altro, non è garantita la completezza del database: nel caso delle cave, così come in quello militare, si è osservata la parzialità dell'informazione presente. I vari database esterni consultati e le segnalazioni presentate ed analizzate sono una testimonianza della varietà dell'informazione pro- cessata e delle difficoltà nel reperire fonti armoniche tra di loro come esemplificato dalle diverse - e non sempre comunicanti - banche dati utilizzate per il caso del dissesto idraulico fluviale.

In definitiva, gli esempi riportati pongono con forza la questione delle possibili sinergie tra sapere contestuale e sapere esperto. Nello specifico si auspica una relazione virtuosa e costruttiva tra i due livelli, cercando di superare le retoriche che caratterizzano e condizionano i processi partecipativi: da parte del sapere esperto o tecnico si dovrebbe tenere in effettiva considerazione quanto emerge dal coinvolgimento degli abitanti e non considerare queste pratiche come atti dovuti. Dall'altro, gli stessi abitanti dovrebbero cercare di affrancarsi da una lettura che consideri solo il problema contingente senza una efficace e problematica contestualizzazione. Questo potrebbe favorire il dialogo e una fruttuosa e genuina integrazione dei saperi.

\section{Ringraziamenti}

Questo contributo è stato reso possibile anche a partire dalle elaborazioni effettuate nell'ambito del progetto dipartimentale PaRiDe "I paesaggi del rischio e del degrado", condotto presso l'Università di Udine. $\mathrm{Al}$ riguardo gli autori desiderano ringraziare i colleghi Mauro Pascolini e Salvatore Amaduzzi.

\section{Riferimenti bibliografici}

Baccichet, M. (2015). Disegno e crisi della pianificazione militare lungo la cortina di ferro: il caso del Friuli Venezia Giulia. In Bacchichet, M. (a cura di). La Fortezza FVG. Dai paesaggi della guerra fredda alle aree militari dismesse. Gorizia, Edicom Edizioni, 11-77.

Balletti, F. (2007). Introduzione. Riflessioni sull'interazione tra sapere tecnico e sapere locale. In Balletti, F. (a cura di). Sapere tecnico - sapere locale. Conoscenza, identificazione, scenari per il progetto. Firenze, Alinea, 11-28.

Bellencin Meneghel, G., Lombardi, D., Zamolo, M. (2003). Le aree dismesse nella città di Udine. In Leone, U. (a cura di). Aree dismesse e verde urbano. Nuovi paesaggi in Italia. Bologna, Patron, 291-332.

Besse, J.M. (2020). Paesaggio e ambiente. Natura, territorio, percezione. Roma, DeriveApprodi.

Bianchetti, A. (2003), Ombre e luci dei processi di dismissione in una universal caserma del secolo breve: il Friuli. In Leone, U. (a cura di). Aree dismesse e verde 
urbano. Nuovi paesaggi in Italia. Bologna, Patron, 349366.

Bianchetti, A., Zamolo, M. (2000). Le aree dismesse in Friuli-Venezia Giulia: un'introduzione alla ricerca. Quaderni della ricerca, dicembre, 93-114.

Bressan, G., Amaduzzi, S. (2020). Map-based surveys for mapping high-quality and degraded sites. In Gallia, A. (a cura di). Territorio: rischio/risorsa. Roma, Labgeo Caraci, 197-213.

Bressan, G., Pascolini, M. (2019). Dalle percezioni della popolazione al dato georiferito: studio quali-quantitativo del paesaggio del Friuli V.G.. In Atti della Conferenza Nazionale di Geomatica e Informazione Territoriale ASITA 2019. Milano, ASITA, 115-122.

Castiglioni, B., De Marchi, M. (a cura di). (2009). Di chi è il paesaggio? La partecipazione degli attori nella individuazione, valutazione e pianificazione. Padova, Cleup.

Castiglioni, B., De Marchi, M., Ferrario, V., Bin, S., Carestiano, N., De Nardi, A. (2010). Il paesaggio "democratico" come chiave interpretativa del rapporto tra popolazione e territorio: applicazioni al caso Veneto. Rivista Geografica Italiana, 117 (1), 93-126.

Castiglioni, B., Ferrario, V. (2020). Sguardi interdisciplinari sul paesaggio alla luce della Convenzione europea, Rivista Geografica Italiana, 127 (4), 191-198.

Coletto, W. (2015), Lesperienza di una cartografia partecipata: la mappa delle aree militari in Friuli. In Bacchichet, M. (a cura di). La Fortezza FVG. Dai paesaggi della guerra fredda alle aree militari dismesse. Gorizia, EdicomEdizioni, 167-170.

Consiglio d'Europa (2000). Convenzione Europea del Paesaggio. http://www.convenzioneeuropeapaesaggio.beniculturali.it/uploads/2010_10_12_11_22_02.pdf

Corde Architetti (2016). Un paese di primule e caserme. Pordenone, Cinemazero.

Cosgrove, D. Petts, G. (a cura di). (1990). Water, Engineering and Landscape. London, Belhaven Press.

De Cillia, A. (2000). I fiumi del Friuli Venezia Giulia. Udine, Gaspari Editore.

Dematteis, G. (2007). Paesaggio come "codice genetico". In Balletti, F. (a cura di). Sapere tecnico - sapere locale. Conoscenza, identificazione, scenari per il progetto. Firenze, Alinea, 33-37.

Democrazia Proletaria (1989). Bella Italia Armate sponde. Roma, Irene edizioni.
Fischer, F. (2000). Citizens, Experts, and the Environment. London, Duke University Press.

Gisotti, G. (2008). Le Cave. Recupero e pianificazione e ambientale. Palermo, Dario Flaccovio Editore.

Goodchild, M.F. (2007). Citizens as sensors: the world of volunteered geography. GeoJournal, 69, 211-221.

Guaran, A. (2003). Aree dismesse in Friuli-Venezia Giulia. Lasse vallivo montano del Canal del Ferro e della Val Canale. In Leone, U. (a cura di). Aree dismesse e verde urbano. Nuovi paesaggi in Italia. Bologna, Patron, 333-366.

Guaran, A., Pascolini, M. (a cura di). (2019). Pianificazione e governo del paesaggio: analisi, strategie, strumenti. Lapporto pluridisciplinare dell'Università di Udine al Piano paesaggistico regionale del Friuli Venezia Giulia. Udine, Forum.

Guarini, R., Tassinari, F. (2000). Statistica economica. Problemi e metodi di analisi. Bologna, Il Mulino.

IRDISP (1984). Quello che i russi già sanno e gli italiani non devono sapere. Roma, Istituto di Ricerche per il Disarmo e lo Sviluppo della Pace, $2^{\text {a }}$ edizione.

Lefebvre, H. (1991). The production of Space. Oxford, Wiley-Blackwell.

Magnaghi, A. (2005). Il ritorno dei luoghi nel progetto. In Magnaghi, A. (a cura di). La rappresentazione identitaria del territorio: atlanti, codici, figure, paradigmi per il progetto locale. Firenze, Alinea, 7-18.

Magnaghi, A. (2020). Il principio territoriale. Torino, Bollati Boringhieri.

Marbach, G. (a cura di). (1991). Statistica economica. Torino, Utet.

Mattioli, C. (2012). I conflitti territoriali: dall'impasse alla durabilità. Planum. The Journal of Urbanism, 25 (2), 1-9.

Michelutti, P. (2012). Servitù militari e militarizzazione. Il Friuli Venezia Giulia 1949-1989. Italia Contemporanea, 267, 291-307.

Minoia, P., Faggi, P. (1995). Gestione delle risorse idriche e dinamiche territoriali. Padova, Università di Padova (Materiali del Dipartimento di Geografia, 17).

Nogué, J. (2017). Paesaggio, Territorio, Società Civile. Il senso del luogo nel contemporaneo. Melfi, Libria.

Papotti, D. (2008). L'approccio geografico al paesaggio: una rilettura del rapporto fra natura e cultura alla luce della Convenzione Europea del Paesaggio. In Teofili, C., Clarino, R. (a cura di). Riconquistare il paesaggio, Roma, WWF, MIUR, 124-138. 
Peccol, E. (2015), Mappare le aree militari dismesse alla luce del contenimento del consumo di suolo. In Bacchichet, M. (a cura di). La Fortezza FVG. Dai paesaggi della guerra fredda alle aree militari dismesse. Gorizia, EdicomEdizioni, 153-166.

Perrone, C. (2011). Per una pianificazione a misura di territorio. Regole insediative, beni comuni e pratiche interattive. Firenze, Firenze University Press.

Petruzzi, R., Petriccione, L. (2019). Costruire ai tempi della Guerra Fredda. L'architettura della fortificazione permanente della frontiera orientale. Udine, Forum.

Pieri Buti, G. (2007). Cave ed ex-cave. Un problema geologico ed ambientale. Geologia dell'Ambiente, 2, 26-27

Poli, D. (2013). Democrazia e pianificazione del paesaggio: governance, saperi contestuali e partecipazione per elevare la coscienza di luogo. Rivista Geografica Italiana, 120 (4), pp. 343-361.

Presidenza della Repubblica Italiana (2004). Decreto Legislativo 22 gennaio 2004, n. 42. Codice dei beni culturali e del paesaggio, ai sensi dell'articolo 10 della legge 6 luglio 2002, n. 137. Gazzetta Ufficiale n. 45 del 24 febbraio 2004 - Supplemento Ordinario n. 28.

Regione Autonoma FVG (2016). Legge regionale 15 luglio 2016, n. 12 Disciplina organica delle attività estrattive. https://lexview-int.regione.fvg.it/fontinormative/xml/xmllex.aspx?anno $=2016 \&$ legge $=12$ (testo vigente).

Regione Autonoma FVG (2018a). Piano paesaggistico regionale del Friuli Venezia Giulia, Relazione generale, Trieste. https://www.regione.fvg.it/rafvg/cms/RAFVG/ ambiente-territorio/pianificazione-gestione-territorio/ FOGLIA21/

Regione Autonoma FVG (2018b). Individuazione delle aree di cava dismesse sul territorio regionale ai sensi dellarticolo 10, comma 4 della legge regionale 15 luglio 2016, n.12, Decreto n. 2542/AMB del 06/07/2018. http:// www.regione.fvg.it/rafvg/export/sites/default/RAFVG/ ambiente-territorio/geologia/FOGLIA15/allegati/Decreto_di_individuazione_delle_aree_di_cava_dismesse.pdf

Roe, M. (2012). Landscape and Participation. Londra, Routledge.

Quaini, M. (2007). Senso comune e/o sapere locale versus sapere scientifico e tecnico. In Balletti, F. (a cura di). Sapere tecnico - sapere locale. Conoscenza, identificazione, scenari per il progetto. Firenze, Alinea, 38-54.

Smith, N. (1984). Uneven development: Nature, Capital and the Production of Space. Oxford, Wiley-Blackwell.
Swyngedouw, E. (2015). Liquid power: Contested hydromodernities in twentieth-century Spain. Cambridge (MA), MIT Press.

Tondelli, S., Conticelli, E. (2012). Il percorso di individuazione del degrado nei paesaggi regionali. https://territorio. regione.emilia-romagna.it/paesaggio/studi-analisi-eapprofondimenti-tematici/paesaggi-degradati (accesso 18 settembre 2021).

Tramontin, L., Pellegrini, P., Astolfo G. (2011). Patussi: premesse per l'uso di una ex caserma. Udine, Forum.

Turri, E. (2014) Semiologia del paesaggio. Venezia, Marsilio.

Vallerani, F. (2004). Acque a nordest. Da paesaggio moderno ai luoghi del tempo libero. Verona, Cierre.

Vallerani, F., Varotto, M. (a cura di). (2005). Il grigio oltre le siepi. Portogruaro, Nuova Dimensione.

Vallerani, F., Visentin, F. (a cura di). (2018). Waterways and the Cultural Landscape. Londra, Routledge.

Varotto, M. (2012) Geografie del declino civico? Il fenomeno dei comitati spontanei in Veneto. Bollettino della Società Geografica Italiana, serie 13, 5 (1), 43-58.

Zaccomer, G.P. (2018). L'analisi territoriale socio-economica in ambito paesaggistico. Gli indicatori compositi per la zonizzazione territoriale del Friuli Venezia Giulia. Udine, Forum. 